\title{
Pullback crossed modules in the category of racks
}

\author{
Kadir Emir*, Hatice Gülsün Akay \\ Department of Mathematics and Computer Science, Eskişehir Osmangazi University, Turkey.
}

\begin{abstract}
In this paper, we define the pullback crossed modules in the category of racks that are mainly based on a pullback diagram of rack morphisms with extra crossed module data on some of its arrows. Furthermore, we prove that the conjugation functor, which is defined between the category of crossed modules of groups and of racks, preserves the pullback crossed modules.
\end{abstract}

Mathematics Subject Classification (2010). 20N02, 18D05, 18A30, 18A40

Keywords. rack, crossed module, limit

\section{Introduction}

A rack $R$ is a set equipped with a non-associative binary operation satisfying:

$$
(x \triangleleft y) \triangleleft z=(x \triangleleft z) \triangleleft(y \triangleleft z)
$$

for all $x, y, z \in R$, and one additional property of this binary operation. Moreover, a rack is called "quandle" if it further satisfies $x \triangleleft x=x$, for all $x \in R$. These total quandle axioms are related to the Reidemeister moves of knot diagrams, and this yields a connection between knot theory and the theory of quandles (hence racks) [9]. Racks have been variously studied under plenty of names and a variety of terminology in literature. They are called automorphic sets [1], crystals [8], left distributive left quasigroups [10] and racks (as a modification of wrack) [4]. The most important example of racks comes from the conjugation in a group $G$ where $g \triangleleft h=h^{-1} g h$, for all $g, h \in G$. This property yields a functor Conj: Grp $\rightarrow$ Rack from the category of groups to the category of racks. Moreover, there exists an adjunction [7] between these two categories with:

$$
\operatorname{Hom}_{\text {Grp }}(\operatorname{As}(X), G) \cong \operatorname{Hom}_{\text {Rack }}(X, \operatorname{Conj}(G)) \text {, }
$$

where the functor As: Rack $\rightarrow$ Grp is left adjoint to the functor Conj.

A crossed module of groups [11] $\mathcal{G}=(\partial: E \rightarrow G, \cdot)$ is defined by a group homomorphism $\partial: E \rightarrow G$, together with a (right) group action of $G$ on $E$ satisfying the Peiffer relations, i.e. $\partial(e \cdot g)=g^{-1} \partial(e) g$ and $f \cdot \partial(e)=e^{-1} f e$, for all $e, f \in E$ and $g \in G$. Crossed modules of racks [5] generalize the notion of crossed modules of groups satisfying two parallel Peiffer conditions. An interesting result of this notion is the functors As and Conj preserving the crossed module structures, see [5]. Therefore, we can also consider them as the (induced) functors between the category of crossed modules of groups XGrp and

\footnotetext{
*Corresponding Author.

Email addresses: kadiremir86@gmail.com (K. Emir), hgulsun@ogu.edu.tr (H. Gülsün Akay)

Received: 20.12.2016; Accepted: 11.10.2017
} 
the category of crossed modules of racks XRack, denoted by $\mathrm{As}^{\star}$ and Conj ${ }^{\star}$ respectively. Then, the previous adjunction leads to the following extended adjunction:

$$
\operatorname{Hom}_{X G r p}\left(\operatorname{As}^{\star}(X), \mathcal{G}\right) \cong \operatorname{Hom}_{X R a c k}\left(X, \operatorname{Conj}^{\star}(\mathcal{G})\right) \text {. }
$$

Consequently, one can say that the functor $\mathrm{Conj}^{\star}$ preserves limits and $\mathrm{As}^{\star}$ preserves colimits.

Crossed modules of groups or racks, which have the same fixed codomain $A$ will be called $\operatorname{crossed}_{A}$ modules, and lead to full subcategories of the corresponding categories. We denote these categories by $\mathrm{XGrp}_{\mathrm{A}}$ and $\mathrm{XRack}_{\mathrm{A}}$, respectively.

Pullback crossed modules in the category of groups are introduced in [3] which is derived originally from [2]. Explicitly, let $\phi: S \rightarrow R$ be a fixed group homomorphism and $\partial: P \rightarrow$ $R$ be a crossed module. Let $A$ be the pullback in the category of groups with the diagram:

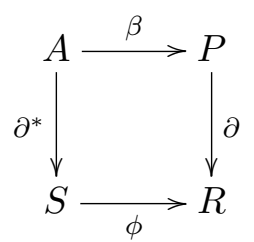

Then, $S$ acts on $A \subset P \times S$ by the rule $a^{s}=\left((\beta a)^{\phi s}, s^{-1}(\partial a) s\right)$ for all $s \in S$ and $a \in A$ that makes $\partial^{*}: A \rightarrow S$ a crossed module and $(\beta, \phi)$ a crossed module morphism. This morphism is universal for morphisms from $\operatorname{crossed}_{R} \operatorname{modules}_{\text {to }} \operatorname{crossed}_{S} \operatorname{modules}$ that induce $\phi: S \rightarrow R$. Writing $A=\phi^{*} P$ we obtain a functor $\phi^{*}: \mathrm{XGrp}_{\mathrm{R}} \rightarrow \mathrm{XGrp}_{\mathrm{S}}$ which is called restriction that is left adjoint to the induced functor introduced in [3].

In this paper, we construct the pullback crossed modules in the category of racks that will generalize the pullback crossed modules of groups. Furthermore, we see that the functor $\mathrm{Conj}^{\star}$ preserves the pullback crossed module structure in the sense of the following commutative diagram:

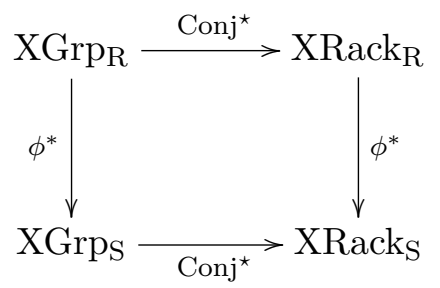

for any arbitrary but fixed group homomorphism $\phi: S \rightarrow R$.

\section{Preliminaries}

We recall some notions from $[5,7]$ that will be used in the sequel.

\subsection{Category of racks}

Definition 2.1. A (right) rack $R$ is a set equipped with a (right) binary operation satisfying the following conditions:

- for each $a, b \in R$, there is a unique $c \in R$ such that:

$$
c \triangleleft a=b,
$$

- for all $a, b, c \in R$, we have:

$$
(a \triangleleft b) \triangleleft c=(a \triangleleft c) \triangleleft(b \triangleleft c) .
$$


A pointed rack is a rack $R$ with an element $1 \in R$ such that (for all $a \in R$ ):

$$
1 \triangleleft a=1 \quad \text { and } \quad a \triangleleft 1=a .
$$

From now on, all racks will be pointed.

Let $R, S$ be two racks. A rack homomorphism is a map $f: R \rightarrow S$ such that:

$$
f(a \triangleleft b)=f(a) \triangleleft f(b) \quad \text { and } \quad f(1)=1,
$$

for all $a, b \in R$. Thus we have the category of racks denoted by Rack. Alternatively, for a point of view on racks where the two right and left rack operations are treated on an equal basis, see [6].

\section{Examples:}

1) Given a group $G$, there exists a rack structure on $G$ where the binary operation is:

$$
g \triangleleft h=h^{-1} g h,
$$

for all $g, h \in G$. This rack is called the conjugation rack of $G$, from which we get the functor:

$$
\text { Conj: Grp } \rightarrow \text { Rack. }
$$

2) The core rack on a group $G$ is defined by:

$$
g \triangleleft h=h g^{-1} h,
$$

for all $g, h \in G$; however this construction is not functorial.

3) Let $P, R$ be two racks, we have a rack structure on $P \times R$ defined by:

$$
(p, r) \triangleleft\left(p^{\prime}, r^{\prime}\right)=\left(p \triangleleft p^{\prime}, r \triangleleft r^{\prime}\right),
$$

which is also the product object in the category of racks.

\subsection{Rack action}

Definition 2.2. Let $R$ be a rack and $X$ be a set. We say that $X$ is an $R$-set when there are bijections $(\cdot r): X \rightarrow X$ for all $r \in R$ such that:

$$
(x \cdot r) \cdot r^{\prime}=\left(x \cdot r^{\prime}\right) \cdot\left(r \triangleleft r^{\prime}\right),
$$

for all $x \in X$ and $r, r^{\prime} \in R$.

Definition 2.3. Let $R$ be a rack and $X$ be an $R$-set. The hemi-semi-direct product $X \rtimes R \subset X \times R$ is the rack defined by:

$$
(x, r) \triangleleft\left(x^{\prime}, r^{\prime}\right)=\left(x \cdot r^{\prime}, r \triangleleft r^{\prime}\right),
$$

for all $x, x^{\prime} \in X$ and $r, r^{\prime} \in R$.

Remark that $x^{\prime}$ disappears in the hemi-semi direct operation which is the main technical difference from the semi-direct product of groups and causes various problems when we deal with it.

Definition 2.4. Let $R, S$ be two racks. We say that $S$ acts on $R$ by automorphisms when there is a (right) action of $S$ on $R$ and:

$$
\left(r \triangleleft r^{\prime}\right) \cdot s=(r \cdot s) \triangleleft\left(r^{\prime} \cdot s\right)
$$

for all $s \in S$ and $r, r^{\prime} \in R$. 


\subsection{Crossed modules of racks}

Definition 2.5. A crossed module of racks $(R, S, \partial)$ is a rack homomorphism $\partial: R \rightarrow S$ together with a (right) rack action of $S$ on $R$ such that following two Peiffer relations hold (for all $r, r^{\prime} \in R$ and $s \in S$ ):

$\mathrm{X} 1) \partial(r \cdot s)=\partial(r) \triangleleft s$,

$\mathrm{X} 2) r \cdot \partial\left(r^{\prime}\right)=r \triangleleft r^{\prime}$.

If $(R, S, \partial)$ and $\left(R^{\prime}, S^{\prime}, \partial^{\prime}\right)$ are two crossed module of racks, a crossed module morphism:

$$
\left(f_{1}, f_{0}\right):(R, S, \partial) \rightarrow\left(R^{\prime}, S^{\prime}, \partial^{\prime}\right)
$$

is a tuple which consists of rack homomorphisms $f_{1}: R \rightarrow R^{\prime}, f_{0}: S \rightarrow S^{\prime}$ such that:

- $\partial^{\prime} f_{1}=f_{0} \partial$,

- $f_{1}(r \cdot s)=f_{1}(r) \cdot f_{0}(s)$,

for all $r \in R, s \in S$. Thus we get the category of crossed modules of racks, denoted by XRack.

\section{Examples:}

1) Let $N \subset R$ be a normal subrack of $R$ (i.e. $n \triangleleft r \in N$ for all $n \in N, r \in R$ ). The inclusion map $N \rightarrow R$ is a crossed module (inclusion crossed module) where the action is defined by the main rack operation.

2) Let $\mu: M \rightarrow N$ be a crossed module of groups. We obtain a crossed module of racks by passing to the associated conjugation racks of $M$ and $N$.

\section{Fiber product of racks}

Definition 3.1. Let $\alpha: P \rightarrow R$ and $\beta: S \rightarrow R$ be two rack homomorphisms. The fiber product $P \times{ }_{R} S$ is the subrack of the rack $P \times S$ defined by:

$$
P \times{ }_{R} S=\{(p, s) \mid \alpha(p)=\beta(s)\} .
$$

From the categorical point of view, the fiber product is the equalizer of the parallel rack homomorphisms:

$$
P \times S \frac{\alpha \circ \pi_{1}}{\beta \circ \pi_{2}} \longrightarrow R
$$

Proposition 3.2. Let $(P, R, \alpha)$ and $(S, R, \beta)$ be two crossed modules of racks. The map $\partial: P \times_{R} S \rightarrow R$ given by:

$$
\partial(p, s)=\alpha(p)=\beta(s)
$$

yields a crossed module $\left(P \times_{R} S, R, \partial\right)$ with the (right) rack action:

$$
\begin{array}{cl}
\left(P \times{ }_{R} S\right) \times R & \rightarrow P \times{ }_{R} S \\
((p, s), r) & \mapsto(p, s) \cdot r=(p \cdot r, s \cdot r)
\end{array}
$$

Proof. The action of $R$ is well-defined, i.e. it preserves $P \times_{R} S$. This follows directly from $\alpha(p) \triangleleft r=\beta(s) \triangleleft r$. Moreover, it satisfies the conditions (2.1) and (2.2) since:

$$
\begin{aligned}
((p, s) \cdot r) \cdot r^{\prime} & =(p \cdot r, s \cdot r) \cdot r^{\prime} \\
& =\left((p \cdot r) \cdot r^{\prime},(s \cdot r) \cdot r^{\prime}\right) \\
& =\left(\left(p \cdot r^{\prime}\right) \cdot\left(r \triangleleft r^{\prime}\right),\left(s \cdot r^{\prime}\right) \cdot\left(r \triangleleft r^{\prime}\right)\right) \\
& =\left(\left(p \cdot r^{\prime}\right),\left(s \cdot r^{\prime}\right)\right) \cdot\left(r \triangleleft r^{\prime}\right) \\
& =\left((p, s) \cdot r^{\prime}\right) \cdot\left(r \triangleleft r^{\prime}\right),
\end{aligned}
$$


and

$$
\begin{aligned}
\left((p, s) \triangleleft\left(p^{\prime}, s^{\prime}\right)\right) \cdot r & =\left(p \triangleleft p^{\prime}, s \triangleleft s^{\prime}\right) \cdot r \\
& =\left(\left(p \triangleleft p^{\prime}\right) \cdot r,\left(s \triangleleft s^{\prime}\right) \cdot r\right) \\
& =\left((p \cdot r) \triangleleft\left(p^{\prime} \cdot r\right),(s \cdot r) \triangleleft\left(s^{\prime} \cdot r\right)\right) \\
& =((p \cdot r),(s \cdot r)) \triangleleft\left(\left(p^{\prime} \cdot r\right),\left(s^{\prime} \cdot r\right)\right) \\
& =((p, s) \cdot r) \triangleleft\left(\left(p^{\prime}, s^{\prime}\right) \cdot r\right),
\end{aligned}
$$

for all $(p, s),\left(p^{\prime}, s^{\prime}\right) \in P \times_{R} S$ and $r, r^{\prime} \in R$.

Also the map $\partial: P \times_{R} S \rightarrow R$ is a rack homomorphism since:

$$
\begin{aligned}
\partial\left((p, s) \triangleleft\left(p^{\prime}, s^{\prime}\right)\right) & =\partial\left(p \triangleleft p^{\prime}, s \triangleleft s^{\prime}\right) \\
& =\alpha\left(p \triangleleft p^{\prime}\right) \\
& =\alpha(p) \triangleleft \alpha\left(p^{\prime}\right) \\
& =\partial(p, s) \triangleleft \partial\left(p^{\prime}, s^{\prime}\right) .
\end{aligned}
$$

Finally $\left(P \times{ }_{R} S, R, \partial\right)$ is a crossed module of racks since:

$\mathrm{X} 1)$

$$
\begin{aligned}
\partial((p, s) \cdot r) & =\partial(p \cdot r, s \cdot r) \\
& =\alpha(p \cdot r) \\
& =\alpha(p) \triangleleft r \quad(\because X 1 \text { condition of } \alpha) \\
& =\partial(p, s) \triangleleft r,
\end{aligned}
$$

$\mathrm{X} 2)$

$$
\begin{aligned}
(p, s) \cdot \partial\left(p^{\prime}, s^{\prime}\right) & =(p, s) \cdot \alpha\left(p^{\prime}\right) \\
& =\left(p \cdot \alpha\left(p^{\prime}\right), s \cdot \alpha\left(p^{\prime}\right)\right) \\
& =\left(p \cdot \alpha\left(p^{\prime}\right), s \cdot \beta\left(s^{\prime}\right)\right) \quad\left(\because \alpha\left(p^{\prime}\right)=\beta\left(s^{\prime}\right)\right) \\
& =\left(p \triangleleft p^{\prime}, s \triangleleft s^{\prime}\right) \quad(\because X 2 \text { condition of } \alpha, \beta) \\
& =(p, s) \triangleleft\left(p^{\prime}, s^{\prime}\right),
\end{aligned}
$$

for all $(p, s),\left(p^{\prime}, s^{\prime}\right) \in P \times_{R} S$ and $r \in R$.

\section{Pullback crossed modules in the category of racks}

\subsection{Idea}

Suppose that we have a crossed module of racks $(P, R, \partial)$ and a rack homomorphism $\phi: S \rightarrow R$. The pullback crossed module of racks:

$$
\phi^{*}(P, R, \partial)=\left(\phi^{*}(P), S, \partial^{*}\right)
$$

is a crossed module of racks satisfying the following universal property:

For a given crossed module morphism of racks:

$$
(f, \phi):(X, S, \mu) \rightarrow(P, R, \partial)
$$

there exists a unique crossed module morphism:

$$
\left(f^{*}, \operatorname{id}_{S}\right):(X, S, \mu) \rightarrow\left(\phi^{*}(P), S, \partial^{*}\right)
$$


which makes the following diagram commutative:

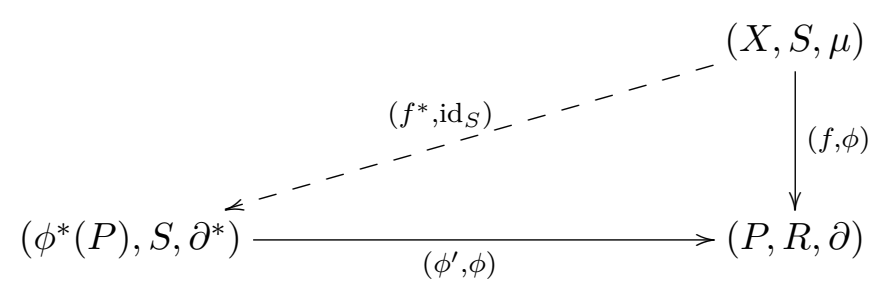

Remark 4.1. In other words, the previous definition can be seen as a pullback of rack homomorphisms:

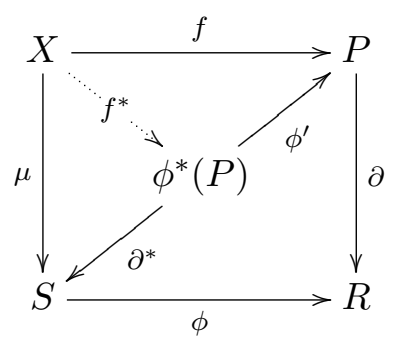

where the arrows $\phi, \phi^{\prime}$ have crossed module structures. It is clear that pullback crossed modules are not the pullback objects in the category XRack.

\subsection{Construction}

Let $(P, R, \partial)$ be a crossed module and let $\phi: S \rightarrow R$ be a rack homomorphism. Define $\phi^{*}(P)=P \times_{R} S$ and $\partial^{*}: \phi^{*}(P) \rightarrow S$ by $\partial^{*}(p, s)=s$. Then $\partial^{*}$ turns into a crossed module where the action of $S$ on $\phi^{*}(P)$ is defined by:

$$
\begin{aligned}
\phi^{*}(P) \times S & \rightarrow \phi^{*}(P) \\
\left((p, s), s^{\prime}\right) & \mapsto(p, s) \cdot s^{\prime}=\left(p \cdot \phi\left(s^{\prime}\right), s \triangleleft s^{\prime}\right)
\end{aligned}
$$

First of all, the action given above is well-defined, i.e. it preserves the set $\phi^{*}(P)$, which follows directly from $\partial(p) \triangleleft \phi\left(s^{\prime}\right)=\phi(s) \triangleleft \phi\left(s^{\prime}\right)$. Moreover, $\partial^{*}$ is a rack homomorphism since:

$$
\begin{aligned}
\partial^{*}\left((p, s) \triangleleft\left(p^{\prime}, s^{\prime}\right)\right) & =\partial^{*}\left(p \triangleleft p^{\prime}, s \triangleleft s^{\prime}\right) \\
& =s \triangleleft s^{\prime} \\
& =\partial^{*}(p, s) \triangleleft \partial^{*}\left(p^{\prime}, s^{\prime}\right),
\end{aligned}
$$

for all $(p, s),\left(p^{\prime}, s^{\prime}\right) \in \phi^{*}(P)$. Furthermore the action conditions are satisfied since:

$$
\begin{aligned}
\left((p, s) \cdot s^{\prime}\right) \cdot s^{\prime \prime} & =\left(p \cdot \phi\left(s^{\prime}\right), s \triangleleft s^{\prime}\right) \cdot s^{\prime \prime} \\
& =\left(\left(p \cdot \phi\left(s^{\prime}\right)\right) \cdot \phi\left(s^{\prime \prime}\right),\left(s \triangleleft s^{\prime}\right) \triangleleft s^{\prime \prime}\right) \\
& =\left(\left(p \cdot \phi\left(s^{\prime \prime}\right)\right) \cdot\left(\phi\left(s^{\prime}\right) \triangleleft \phi\left(s^{\prime \prime}\right)\right),\left(s \triangleleft s^{\prime \prime}\right) \triangleleft\left(s^{\prime} \triangleleft s^{\prime \prime}\right)\right) \\
& =\left(\left(p \cdot \phi\left(s^{\prime \prime}\right)\right) \cdot \phi\left(s^{\prime} \triangleleft s^{\prime \prime}\right),\left(s \triangleleft s^{\prime \prime}\right) \triangleleft\left(s^{\prime} \triangleleft s^{\prime \prime}\right)\right) \\
& =\left(p \cdot \phi\left(s^{\prime \prime}\right), s \triangleleft s^{\prime \prime}\right) \cdot\left(s^{\prime} \triangleleft s^{\prime \prime}\right) \\
& =\left((p, s) \cdot s^{\prime \prime}\right) \cdot\left(s^{\prime} \triangleleft s^{\prime \prime}\right)
\end{aligned}
$$


and

$$
\begin{aligned}
\left((p, s) \triangleleft\left(p^{\prime}, s^{\prime}\right)\right) \cdot s^{\prime \prime} & =\left(p \triangleleft p^{\prime}, s \triangleleft s^{\prime}\right) \cdot s^{\prime \prime} \\
& =\left(\left(p \triangleleft p^{\prime}\right) \cdot \phi\left(s^{\prime \prime}\right),\left(s \triangleleft s^{\prime}\right) \triangleleft s^{\prime \prime}\right) \\
& =\left(\left(p \cdot \phi\left(s^{\prime \prime}\right) \triangleleft p^{\prime} \cdot \phi\left(s^{\prime \prime}\right)\right),\left(s \triangleleft s^{\prime \prime}\right) \triangleleft\left(s^{\prime} \triangleleft s^{\prime \prime}\right)\right) \\
& =\left(p \cdot \phi\left(s^{\prime \prime}\right),\left(s \triangleleft s^{\prime \prime}\right)\right) \triangleleft\left(p^{\prime} \cdot \phi\left(s^{\prime \prime}\right),\left(s^{\prime} \triangleleft s^{\prime \prime}\right)\right) \\
& =\left((p, s) \cdot s^{\prime \prime}\right) \triangleleft\left(\left(p^{\prime}, s^{\prime}\right) \cdot s^{\prime \prime}\right)
\end{aligned}
$$

for all $(p, s),\left(p^{\prime}, s^{\prime}\right) \in \phi^{*}(P)$ and $s^{\prime \prime} \in S$.

Finally $\partial^{*}$ is a crossed module:

$\mathrm{X} 1)$

$$
\begin{aligned}
\partial^{*}\left((p, s) \cdot s^{\prime}\right) & =\partial^{*}\left(p \cdot \phi\left(s^{\prime}\right), s \triangleleft s^{\prime}\right) \\
& =s \triangleleft s^{\prime} \\
& =\partial^{*}(p, s) \triangleleft s^{\prime}
\end{aligned}
$$

$\mathrm{X} 2)$

$$
\begin{aligned}
(p, s) \cdot \partial^{*}\left(p^{\prime}, s^{\prime}\right) & =(p, s) \cdot s^{\prime} \\
& =\left(p \cdot \phi\left(s^{\prime}\right), s \triangleleft s^{\prime}\right) \\
& =\left(p \cdot \partial\left(p^{\prime}\right), s \triangleleft s^{\prime}\right) \quad\left(\because \partial\left(p^{\prime}\right)=\phi\left(s^{\prime}\right)\right) \\
& =\left(p \triangleleft p^{\prime}, s \triangleleft s^{\prime}\right) \quad(\because X 2 \text { condition of } \partial) \\
& =(p, s) \triangleleft\left(p^{\prime}, s^{\prime}\right)
\end{aligned}
$$

for all $(p, s),\left(p^{\prime}, s^{\prime}\right) \in \phi^{*}(P)$.

Furthermore, this construction satisfies the universal property. To state it, we need the crossed module morphism:

$$
\left(\phi^{\prime}, \phi\right):\left(\phi^{*}(P), S, \partial^{*}\right) \rightarrow(P, R, \partial)
$$

where $\phi^{\prime}: \phi^{*}(P) \rightarrow P$ is given by $\phi^{\prime}(p, s)=p$.

Suppose that $(X, S, \mu)$ is an arbitrary crossed module with a crossed module morphism:

$$
(f, \phi):(X, S, \mu) \rightarrow(P, R, \partial)
$$

We need to prove that there exists a unique crossed module morphism:

$$
\left(f^{*}, \operatorname{id}_{S}\right):(X, S, \mu) \rightarrow\left(\phi^{*}(P), S, \partial^{*}\right)
$$

such that:

$$
\left(\phi^{\prime}, \phi\right)\left(f^{*}, \mathrm{id}_{S}\right)=(f, \phi) .
$$

Define $f^{*}: X \rightarrow \phi^{*}(P)$ by $f^{*}(x)=(f(x), \mu(x))$, for all $x \in X$. Then the tuple $\left(f^{*}, \operatorname{id}_{S}\right)$ becomes a crossed module morphism, since (for all $s \in S$ and $x \in X$ ):

$$
\begin{aligned}
f^{*}(x \cdot s) & =(f(x \cdot s), \mu(x \cdot s)) \\
& =(f(x) \cdot \phi(s), \mu(x \cdot s)) \quad(\because(f, \phi) \text { crossed module morphism }) \\
& =(f(x) \cdot \phi(s), \mu(x) \triangleleft s) \quad(\because X 1 \text { condition of } \mu) \\
& =(f(x), \mu(x)) \cdot s \\
& =f^{*}(x) \cdot \operatorname{id}_{S}(s)
\end{aligned}
$$


and

$$
\begin{aligned}
\partial^{*} f^{*}(x) & =\partial^{*}(f(x), \mu(x)) \\
& =\mu(x) \\
& =\operatorname{id}_{S} \mu(x) .
\end{aligned}
$$

Finally the diagram (4.1) commutes, since (for all $x \in X$ ):

$$
\begin{aligned}
\partial^{*} f^{*}(x) & =\partial^{*}(f(x), \mu(x)) \\
& =\mu(x) \\
\phi^{\prime} f^{*}(x) & =\phi^{\prime}(f(x), \mu(x)) \\
& =f(x)
\end{aligned}
$$

and also $\phi \partial^{*}=\partial \phi^{\prime}$ by the definition of $\phi^{*}(P)$.

Let $\left(f^{\prime}, \operatorname{id}_{S}\right):(X, S, \mu) \rightarrow\left(\phi^{*}(P), S, \partial^{*}\right)$ be a crossed module morphism of racks with the same property as $\left(f^{*}, \operatorname{id}_{S}\right)$. Define $p$ and $s$ by $f^{\prime}(x)=(p, s)$. Then we get:

$$
\begin{aligned}
\phi^{\prime} f^{\prime}(x) & =f(x) \Leftrightarrow \phi^{\prime}(p, s)=f(x) \Leftrightarrow p=f(x) \\
\partial^{*} f^{\prime}(x) & =\mu(x) \Leftrightarrow \partial^{*}(p, s)=\mu(x) \Leftrightarrow s=\mu(x)
\end{aligned}
$$

leading to:

$$
f^{\prime}(x)=(p, s)=(f(x), \mu(x))=f^{*}(x)
$$

which implies that $\left(f^{*}, \operatorname{id}_{S}\right)$ is unique and completes the construction.

Definition 4.2. Let us fix a rack $R$ as a codomain for all crossed modules and construct the related category which is the full subcategory of XRack. These kinds of crossed modules will be called as $\operatorname{crossed}_{R}$ modules and denote the corresponding category by $\mathrm{XRack}_{\mathrm{R}}$.

Corollary 4.3. As a consequence of the pullback crossed module structure in the category of racks, we have the functor:

$$
\phi^{*}: \mathrm{XRack}_{\mathrm{R}} \rightarrow \mathrm{XRack}_{\mathrm{S}} .
$$

Example 4.4. Let $\partial: N \rightarrow R$ be an inclusion crossed module and $\phi: S \rightarrow R$ be a rack homomorphism. Then the pullback crossed module is defined by:

$$
\begin{aligned}
\phi^{*}(N) & =\{(n, s) \mid \partial(n)=\phi(s), n \in N, s \in S\} \\
\cong & \{s \in S \mid \phi(s)=n, n \in N\} \\
& =\phi^{-1}(N)
\end{aligned}
$$

with the following commutative diagram:

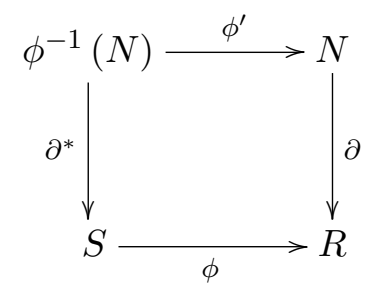

where the preimage $\phi^{-1}(N)$ is a normal subrack of $S$.

It follows that:

Example 4.5. If $N=\{1\}$ and $R$ is a rack, then:

$$
\phi^{*}(\{1\}) \cong\{s \in S \mid \phi(s)=1\}=\operatorname{ker} \phi .
$$

Thus $\left(\operatorname{ker} \phi, S, \partial^{*}\right)$ is a pullback crossed module which implies ker $\phi$ is a normal subrack. 
Corollary 4.6. The kernel of a rack homomorphism is a particular case of a pullback crossed module.

Example 4.7. If $N=R$ and $\phi$ is surjective, then:

$$
\phi^{*}(R)=R \times S .
$$

\section{Functorial approach}

Let $R$ be a rack. The associated group $A s(R)$ is the quotient of the free group $F(R)$ by the normal subgroup generated by the elements $y^{-1} x^{-1} y(x \triangleleft y)$ for all $x, y \in R$, see [7]. This property leads to the functor:

$$
\text { As: Rack } \rightarrow \text { Grp, }
$$

which is left adjoint to the functor Conj.

The major property of these functors is; they both preserve the crossed module structure that is proven in [5]. Consequently:

Corollary 5.1. We have the functors:

$$
\mathrm{As}^{\star}: \text { XRack } \rightarrow \text { XGrp } \quad \text { Conj }^{\star}: \text { XGrp } \rightarrow \text { XRack, }
$$

which are induced by As and Conj, respectively.

Theorem 5.2. There exists an adjunction between the categories of crossed modules of racks and of crossed modules of groups:

$$
\operatorname{Hom}_{X G r p}\left(\operatorname{As}^{\star}(X), \mathcal{G}\right) \cong \operatorname{Hom}_{X R a c k}\left(X, \operatorname{Conj}^{\star}(\mathcal{G})\right),
$$

which is induced by

$$
\operatorname{Hom}_{\text {Grp }}(\operatorname{As}(X), G) \cong \operatorname{Hom}_{\text {Rack }}(X, \operatorname{Conj}(G)) .
$$

Proof. Let $X$ be a rack and $G$ be a group. We know from [7] that; for a given rack homomorphism $f: X \rightarrow \operatorname{Conj}(\mathrm{G})$, there exists a unique group homomorphism $f_{\sharp}$ : As $(\mathrm{X}) \rightarrow \mathrm{G}$ such that the following diagram commutes:

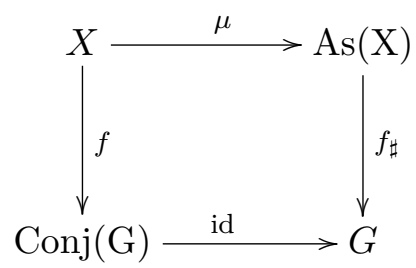

where $\mu$ is the natural map. This diagram leads to (5.1).

One level further, let $X$ be a crossed module of racks and $\mathcal{G}$ be a crossed module of groups. Given a crossed module morphism of racks $(f, g): X \rightarrow \operatorname{Conj}^{\star}(\mathcal{G})$, there exists a unique crossed module morphism of groups $\left(f_{\sharp}, g_{\sharp}\right): \mathrm{As}^{\star}(X) \rightarrow \mathcal{G}$ such that the following diagram commutes:

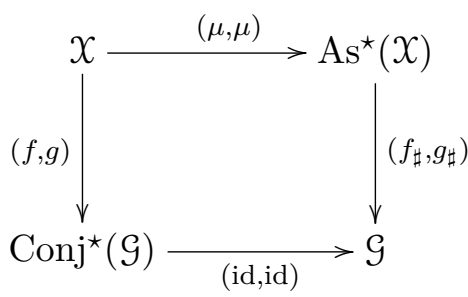

which induces two forms of (4.1) based on rack homomorphisms $f, g$ and proves the adjunction (5.2).

As another main outcome of the paper, we have the following: 
Theorem 5.3. We have the following commutative diagram:

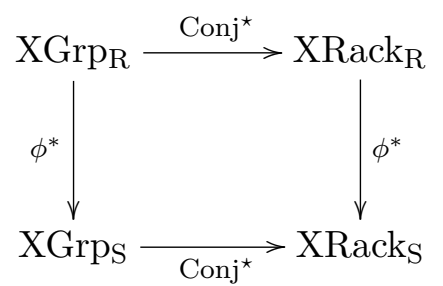

Proof. It follows at once from the known fact that, Conj preserves limits and As preserves colimits since the adjunction (5.2), see also Remark 4.1.

Acknowledgment. The authors are thankful to Friedrich Wagemann and Ronald Brown for their kind assistance through invaluable discussions, and also the anonymous referees for their useful comments that helped improve the paper.

\section{References}

[1] E. Brieskorn, Automorphic sets and braids and singularities, Braids, AMS-IMS-SIAM Jt. Summer Res. Conf., Santa Cruz/Calif. 1986, Contemp. Math. 78, 45-115, 1988.

[2] R. Brown and P. J. Higgins, On the connection between the second relative homotopy groups of some related spaces, Proc. Lond. Math. Soc. 36 (3), 192-212, 1978.

[3] R. Brown and C. D. Wensley, On finite induced crossed modules and the homotopy 2-type of mapping cones, Theory Appl. Categ. 1, 57-71, 1995.

[4] J. Conway and G. Wraith, Unpublished correspondence, 1959.

[5] A. S. Crans and F. Wagemann, Crossed modules of racks, Homology Homotopy Appl. 16 (2), 85-106, 2014.

[6] J. Faria Martins and R. Picken, Link invariants from finite categorical groups, Homology Homotopy Appl. 17 (2), 205-233, 2015.

[7] R. Fenn and C. Rourke, Racks and links in codimension two, J. Knot Theory Ramifications, 1 (4), 343-406, 1992.

[8] L. Kauffman, Knots and Physics, K \& E series on knots and everything, World Scientific, 2001.

[9] S. Nelson, What is...a quandle?, Notices of AMS, 63, 378-380, 2016.

[10] D. Stanovský, Left distributive left quasigroup, Ph.D. thesis, Charles University, Prague, 2004.

[11] J. H. C. Whitehead, On adding relations to homotopy groups, Ann. Math. 42 (2), 409-428, 1941. 